\title{
AN EVALUATION METHOD OF RECORDING CHARACTERISTICS OF THE LIGHT INTENSITY MODULATION DIRECT OVERWRITE (DOW)
}

\author{
Masaya ITO*, Yoshiyuki NAKAKI, Kazuhiko TSUTSUMI and Osamu ITO* \\ *Industrial Electronics \& Systems Lab., \\ Materials \& Electronic Devices Lab., Mitsubishi Electric Corporation \\ 8-1-1 Tsukaguchi-honmachi, Amagasaki, Hyogo, 661, JAPAN
}

\begin{abstract}
The novel evaluation method of recording characteristics has been realized for the light intensity modulation (LIM) DOW using 4-layer MO media. The recording characteristics has been grasped as the read-back signal level on the two dimensional overwrite power plane. Analyzing the distribution of these levels, the power margin of overwriting has been made clear and the permissible power region to be overwritten and the evaluation method of the optimal recording power are defined and estimated.
\end{abstract}

KEYWORDS: LIGHT INTENSITY MODULATION DOW, POWER MARGIN, OPTIMAL RECORDING POWER.

\section{INTRODUCTION}

The light intensity modulation (LIM) DOW is regarded as one of the most promising methods. In particular, LIM-DOW by using 4-layer MO media [1] is suitable for the simplification of the drive because of the fixed bias magnetic field. However, since two laser powers are required for the recording, the power margin is reduced. It is necessary to decide optimal recording power by the test recording of the drive itself. In this paper, the recording process is characterized by the contour of the read signal levels on the two dimensional power plane, and the power margin area is estimated, finally the optimal recording power is evaluated.

\section{THE PROCESS OF LIM DOW OF 4LAYER MO MEDIA}

Fig. 1 shows the overwriting process on the 4-layer MO media by two threshold temperatures, which are the writing layer Curie temperature $\mathrm{Tc}_{2}$ and the memory layer Curie temperature $\mathrm{Tc1}$. $\mathrm{Tc}_{2}$ is the threshold of writing or not writing in the writing layer. $T c_{1}$ is the threshold of copying from the writing layer to the memory layer. If the induced temperature is above Tc2, then the mark (data 1, hereafter called 1recording. ), is normally written in the writing layer by means of the bias magnetic field. In the case of erasing (writing the space or data 0 , so defined as 0 recording. ), the temperature must be between $\mathrm{Tc} 2$ and Tc1. For this temperature, the magnetization of the writing layer still remains at the initial state by the exchange coupling of the initializing layer and the switching layer. These temperatures are generated by the combination of a laser diode power $\mathrm{Ph}$ (higher power) and Pl (lower power).

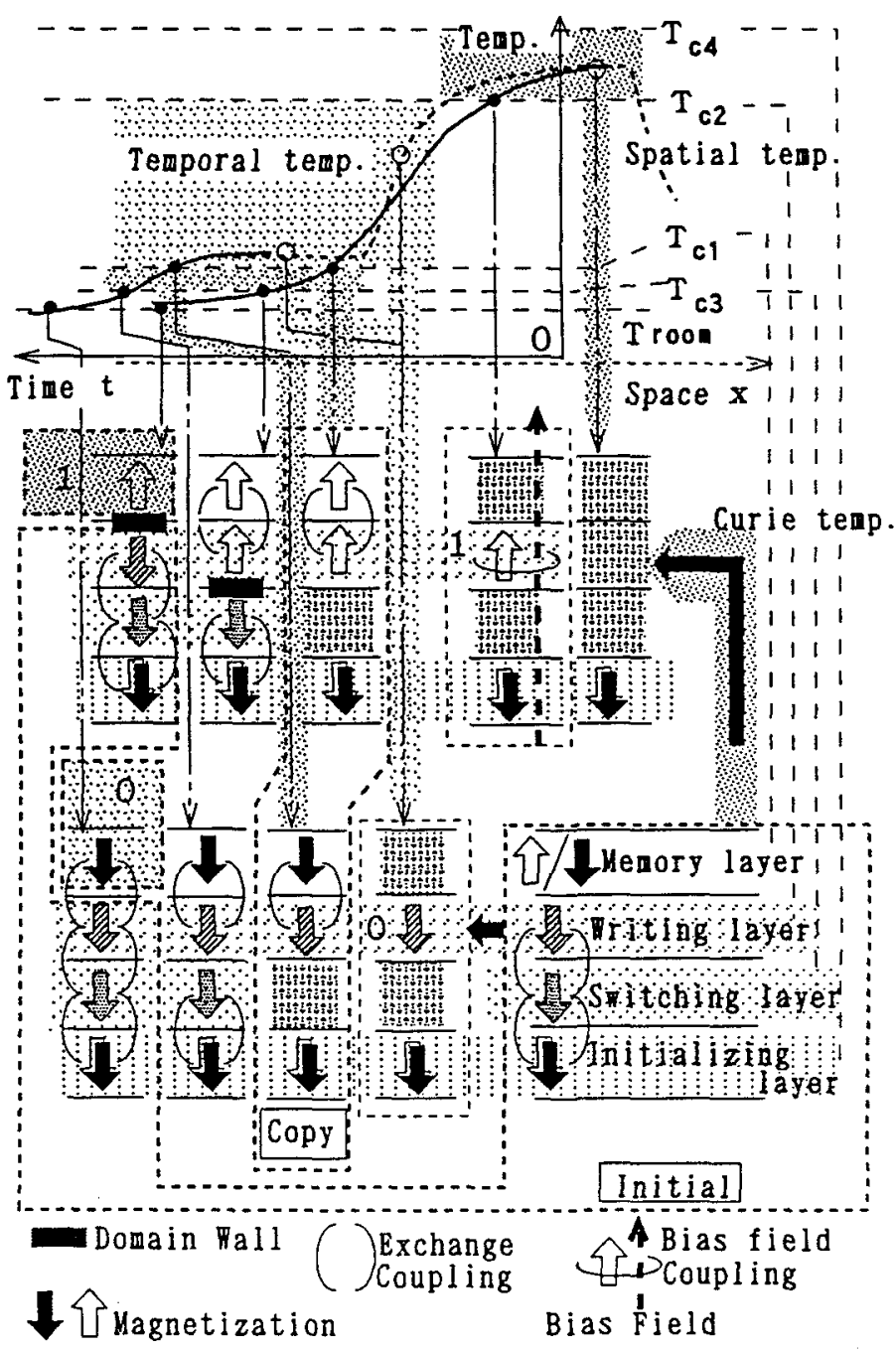

Fig. 1 The process of direct overwrite using the LIM 4-layer MO. 


\section{EVALUATION OF RECORDING CHARACTERISTICS}

For the media, there are three states; copying below $\mathrm{Tc}$, 0 -recording between $\mathrm{T} \mathrm{c}_{1}$ and $\mathrm{Tc}$, and 1-recording above Tc2. The recording power is set to the two levels of $\mathrm{Ph}$ and PI. As the result, six sub-areas exist in the $\mathrm{Ph}-\mathrm{Pl}$ power plane. The correspondence of the power to the temperature is shown and besides, read signal levels also shown schematically in Fig. 2. The boundaries separating these areas are given as four lines from the equation (1) [2].

$$
\begin{aligned}
& l_{1}: \frac{\alpha_{1}}{1-\operatorname{erf}\left(\frac{\beta L}{2}\right)}+k=1, \quad l_{2}: \frac{\alpha_{1}}{\operatorname{erf}\left(\frac{\beta S}{2}\right)}+k=1, \\
& l_{3}: \frac{\alpha_{2}}{1-\operatorname{erf}\left(\frac{\beta L}{2}\right)}+k=1, \quad l_{4}: \frac{\alpha_{2}}{\operatorname{erf}\left(\frac{\beta S}{2}\right)}+k=1, \\
& \alpha_{i}=1-\frac{\eta_{i}}{P_{h}}, \quad \eta_{i} \propto T_{c i}, \quad(i=1,2), \\
& k=\frac{P_{l}}{P_{h}}, \text { error function ; erf }(x)=\frac{2}{\sqrt{\pi}} \int_{0}^{x} e^{-t^{2}} d t, \\
& \text { where } \beta=1 /(\sqrt{2} \sigma), \sigma=\text { spot size, } \\
& L=v \tau_{1}, S=v \tau_{2}, v=\text { linear velocity, } \\
& \tau_{1}=\text { duration of } P_{h}, \tau_{2}=\text { duration of } P_{l} .
\end{aligned}
$$

The size of a recorded mark is proportional to the laser power. Therefore, the read signal level is considered as the good performance index of dynamical 1- or 0-recording. The evaluation of the recording process can be carried out by the following procedure using the test-writing with suitable test patterns. In Table 1, the conditions and the purposes for the test writing are summarized. The characteristics of 1-recording are equivalent to the non-overwrite recording. Obtaining the upper limit of $\mathrm{Ph}$, after initializing by the highest density pattern, the same pattern is recorded at various power combinations of $\mathrm{Ph}$ and $\mathrm{Pl}$, and the signal level is read. On the other hand, since the 0-recording is essentially the ability of copying from the writing layer to the memory layer, and in this process the marks are simultaneously transferred to the memory layer, the characteristics must 'be obtained without the influence of the higher power $\mathrm{Ph}$. This is performed by the reverse recording procedure that the memory layer is initiated by the pattern DC" 1 " and then spaces are reversely written as the 0 -recording by the most sparse pattern at power $\mathrm{Ph}$ instead of P. The read signal level of the 0 -recording is similarly measured as normally recorded marks. That is certainly the superior merit to any other evaluation because of the simplicity and the accuracy.
Table 1. Test-writing conditions

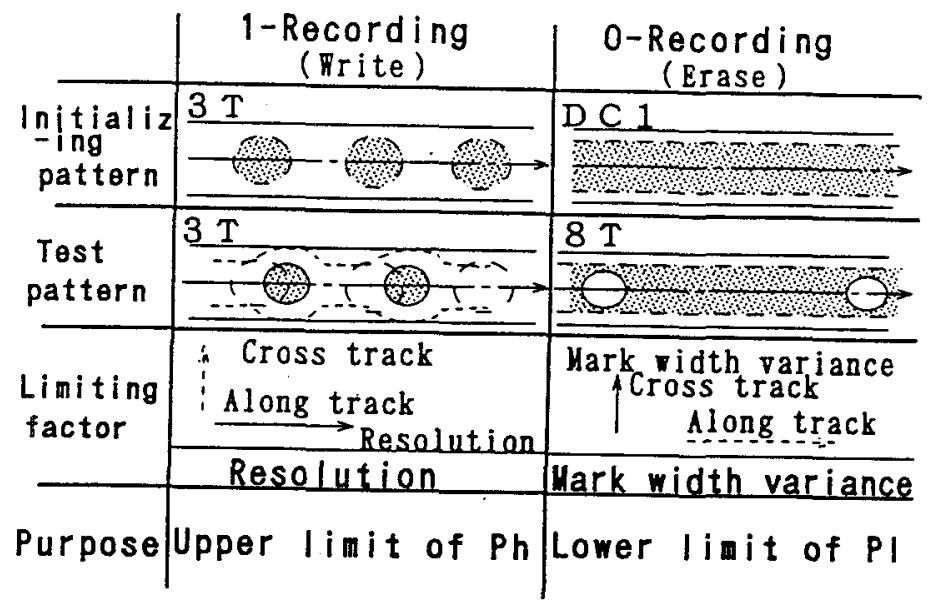

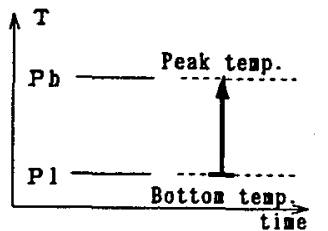

(a) DC duration teep.

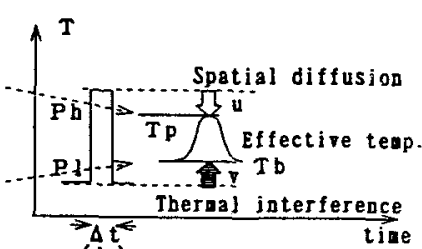

(b) AC duration temp.

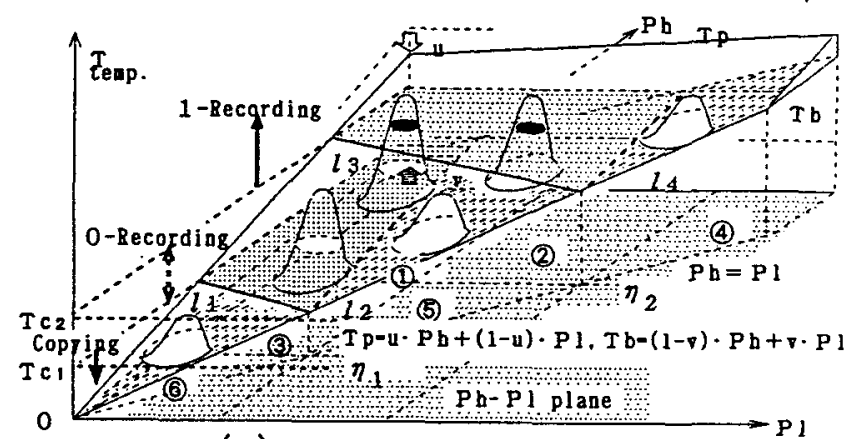

(c) Dow power and temperature

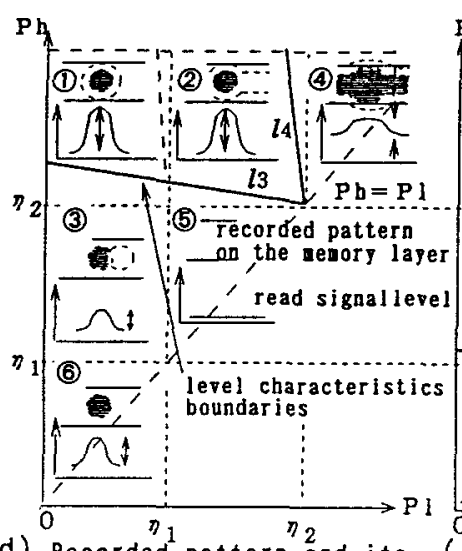

(d) Recorded pattern and its signal levels at the initial $3 T$ recording

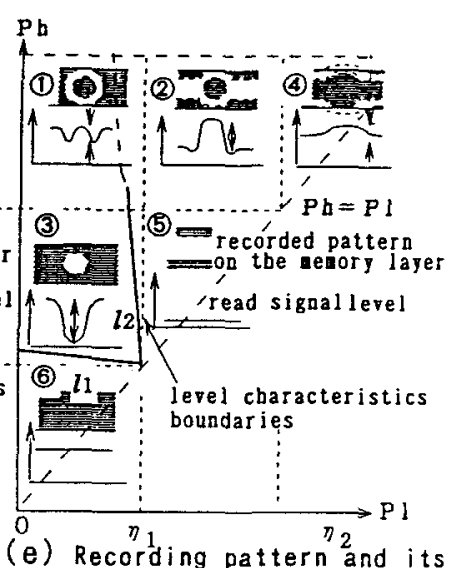

signal levels at the initial DC- I" recording

Fig. 2 Recording operators, six sub areas, the recorded patterns and read signal levels. 
Since the DOW is the process that 1 - and 0 -recording concurrently occurs, it should be indicated by the both areas characterized by the 1 - and 0 -recording on the $\mathrm{Ph}-\mathrm{Pl}$ power plane. This common area is shown in Fig. 3. The line BExT is the linear extension of the boundary between area(3) and area(5) in the 0-record-

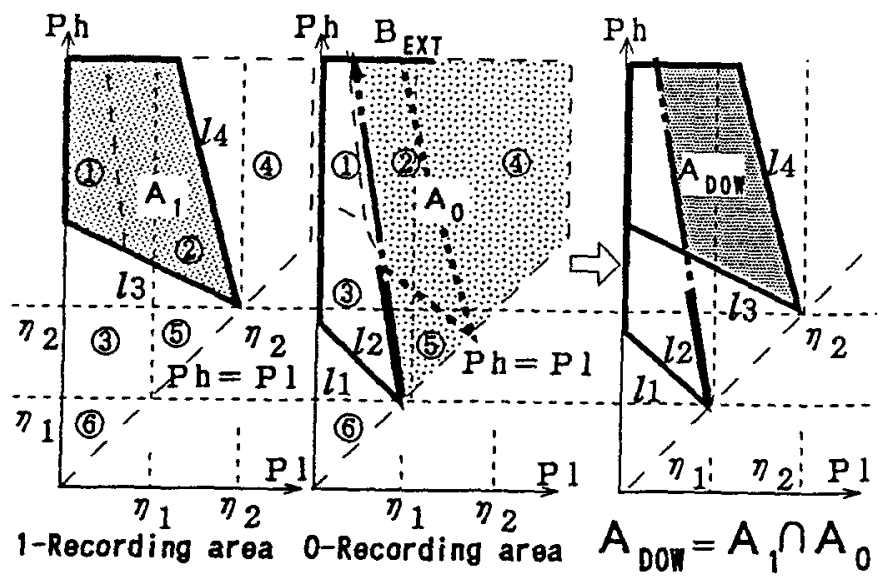

Fig. 3 DOW area in the power plane

ing area. The right side of BEXT is considered as the sufficient erasure i.e. 0 -recording. As illustrated in Fig. 4, three intersected lines on the power plane are plotted, $l s s, l c$, loe. Along these lines, the read signal levels are distributed as shown in Fig. 4, respectively.

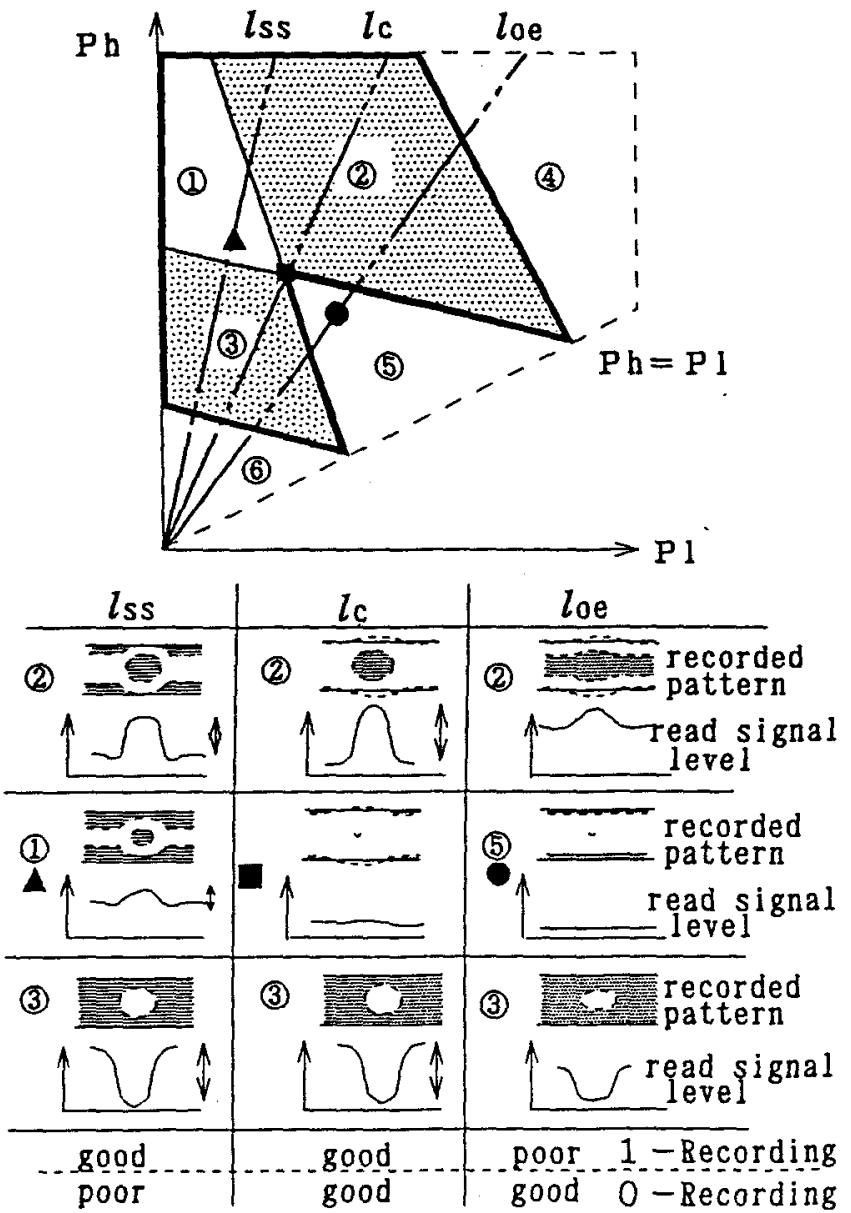

Fig. 4 Optimal power combination
From this level distribution, it is clarified that the best power combination must be on the line $l c$. The permissible area to set independently $\mathrm{Ph}$ and $\mathrm{Pl}$ is described as a rectangle internally touched to ADOw shown in Fig. 3, i.e. a region of overwritable. Since BEXT and boundaries $l_{3}, l_{4}$ manifest the definite threshold, when the power margin is defined as the window at the specified value (such as BER $10^{-5}$ ), then this rectangle must be shrunk to a certain extent (BER $10^{-5}$ corresponds to $43 \%$ ). These regions are shown in Fig. 5. The optimal power Po (Pho, $\mathrm{Plo}$ ) is

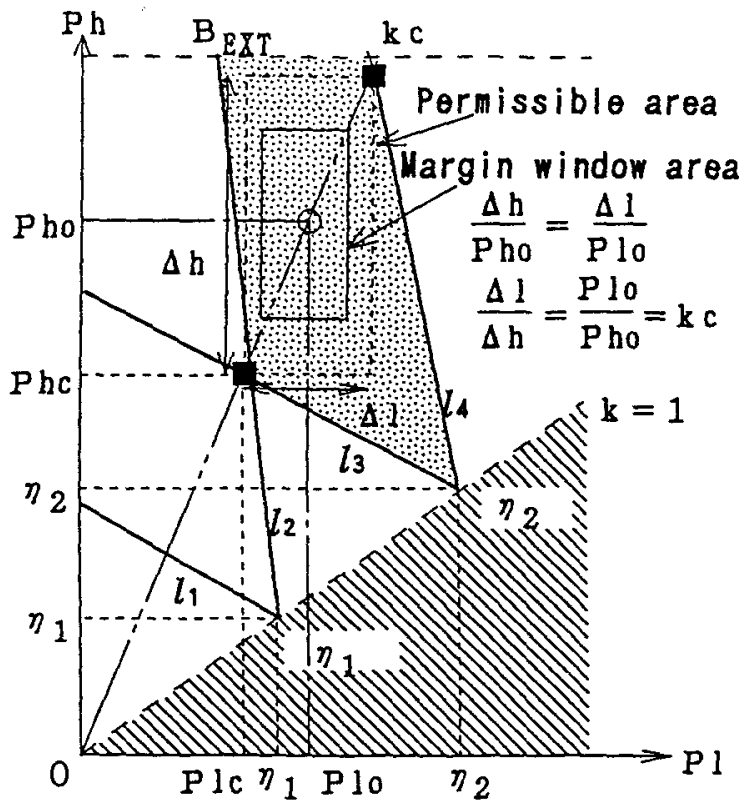

Fig. 5 Optimum DOW power Po and margin window

located at the center of this rectangle, because of the coincidence of the both centers. In particular, the center is independent of the window size at the specified level as above.

\section{RESULTS AND DISCUSSIONS}

As an example, the $(2,7)$ RLL-PPM patterns were recorded at the inner radius. Fig. 6 shows the read signal level contours of $8 \mathrm{~T}$ pattern such that $\mathrm{L} / \mathrm{S}=1 / 11$ for the 0-recording. Fig. 7 is the characteristics of 3T pattern such that $\mathrm{L} / \mathrm{S}=1 / 4$ for the 1 -recording. According to the equation (1), boundaries are calculated as follows,

$$
\begin{array}{ll}
l_{1}: \frac{\alpha_{1}}{0.615}+k=1, & l_{2}: \frac{\alpha_{1}}{0.983}+k=1, \\
l_{3}: \frac{\alpha_{2}}{0.615}+k=1, & l_{4}: \frac{\alpha_{2}}{0.983}+k=1, \\
\text { where } \quad \alpha_{1}=1-\frac{2.8}{P_{h}}, & \alpha_{2}=1-\frac{5.6}{P_{h}} .
\end{array}
$$




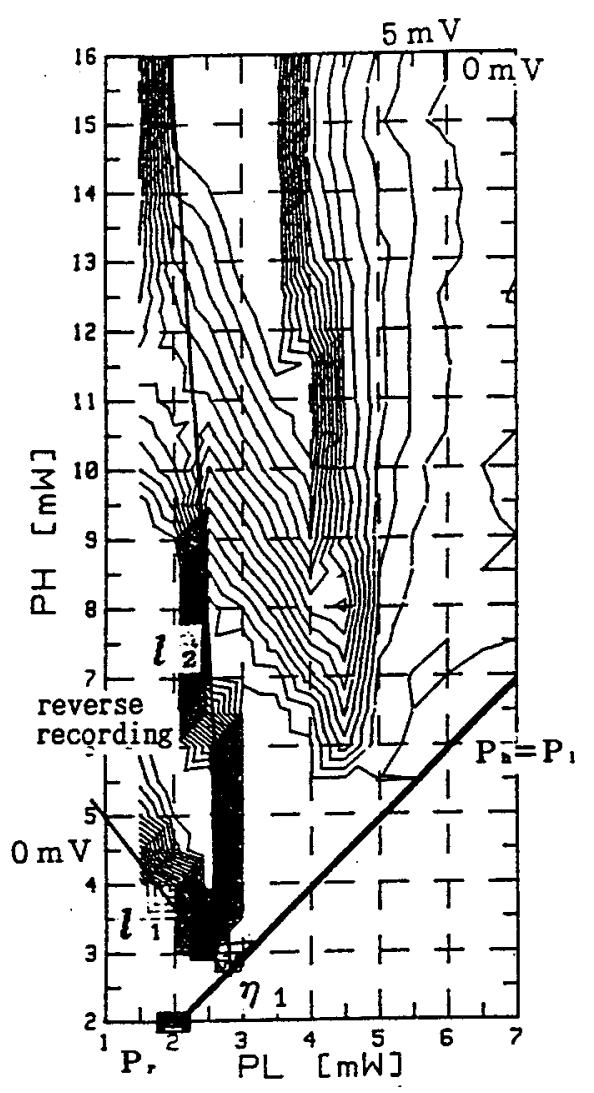

Fig. 6 0-recording characteristics

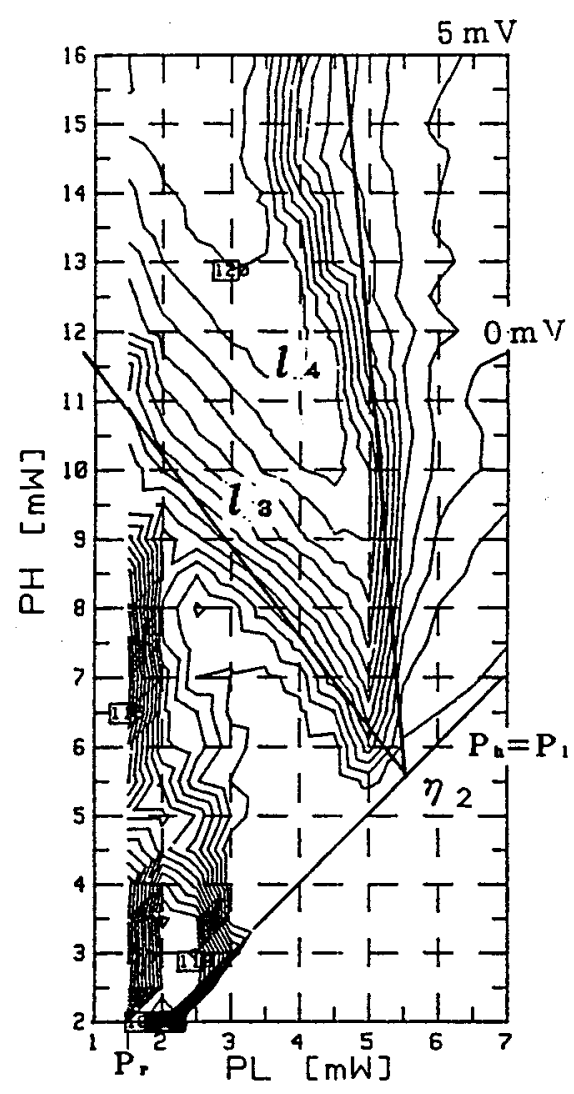

Fig. 7 1-recording characteristics

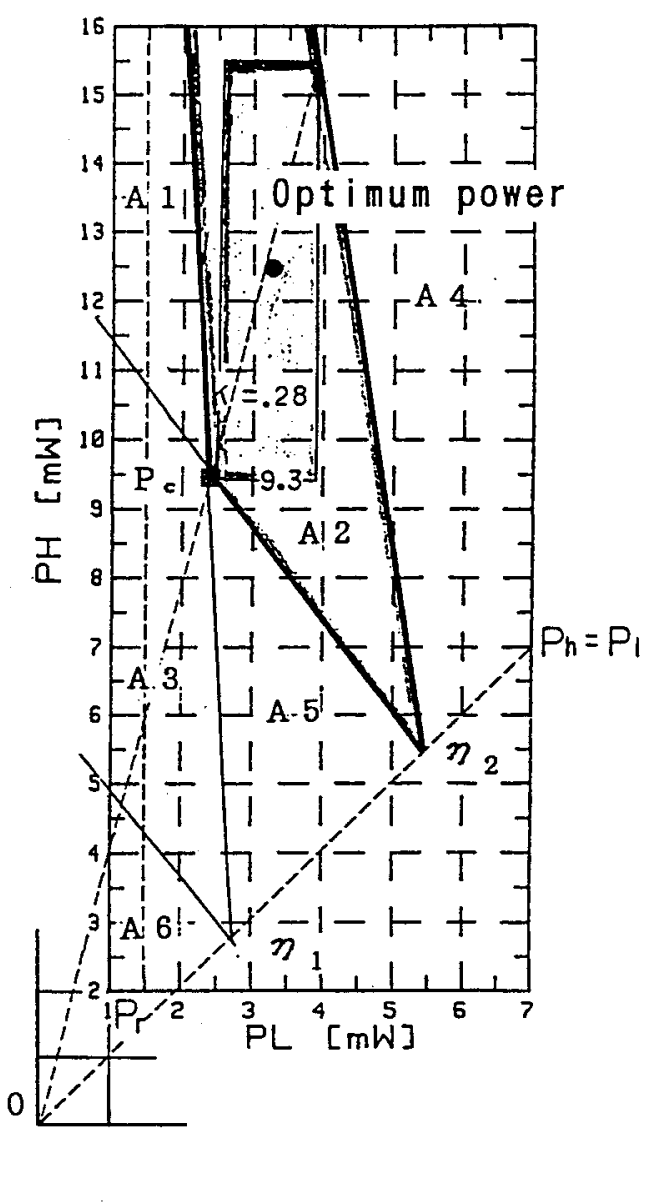

Fig. 8 Optimum power

\section{CONCLUSION}

Fig. 6 and Fig. 7 show measured contours and boundaries respectively. The cross point $\mathrm{Pc}(\mathrm{Phc}, \mathrm{Plc})$ where $l_{2}$ meets $l_{3}$ only depends on the ratio of $\mathrm{Tc}_{2} /$ Tc1. This ratio is normally designed as 2 , then

$$
\begin{aligned}
& k=\frac{P_{L c}}{P_{h c}}=\frac{0.385}{\frac{\eta_{2}}{\eta_{1}}-1+0.385}=0.28, \\
& P_{h c}=93 .
\end{aligned}
$$

These 4 lines and 6 areas are shown in Fig. 8. Note that the calculation results closely correspond to the actual measurement. $A_{1}$ is the self-sharpening area, $A_{2}$ is the overwriting, $A 3$ is the reverse recording, $A 4, A 5$ and $A 6$ are irrelevant areas. In the area $A_{2}$, the rectangle touched to $\mathrm{Pc}$ and the boundaries and the center are obtained respectively. The optimal power $\mathrm{Po}$ (Pho,Plo) is $(12.3 \mathrm{~mW}, 3.5 \mathrm{~mW})$ in this case.
A novel evaluation method for LIM DOW has been developed. Plotting the read-back signal on the power plane, and only monitoring the signal level, the 0 -level boundaries as the threshold of overwriting and the optimal overwrite power have been rapidly calculated from the test-writing condition and the measured read signal level by the drive itself. The results of the calculation by this method is closely correspondent to the adequate overwrite power identified with the measured level contours. Accordingly, the media evaluation could have been also made by the drive itself. This evaluation by self test-writing is very useful for the drive in the point that the estimated optimal power is independent of the variance of media sensitivity.

\section{REFERENCES}

[1] T. Fukami, Y. Nakaki, T. Tokunaga, M. Taguchi, and K. Tsutsumi: J. Appl. Phys., 67, 9, pp. 4415-4416 (1990).

[2] B. Bartholomeusz, D. Genova, and D. Stinson : Appl. Opt. , 29, 20, pp. 3030-3039 (1990). 